\title{
Photoreactive composite coating with composition dependent wetting properties
}

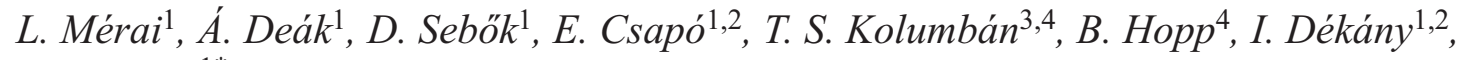 \\ L. Janovák $k^{1 *}$ \\ ${ }^{1}$ Department of Physical Chemistry and Materials Science, University of Szeged, H-6720 Szeged, Rerrich B. tér 1, \\ Hungary \\ ${ }^{2}$ MTA-SZTE Biomimetic Systems Research Group, University of Szeged, H-672, Szeged, Dóm tér 8, Hungary \\ ${ }^{3}$ MTA-SZTE Research Group on Photoacoustic Spectroscopy, University of Szeged, H-6720 Szeged, Dóm tér 9, Hungary \\ ${ }^{4}$ Department of Optics and Quantum Electronics, University of Szeged, H-6720 Szeged, Dóm tér 9, Hungary
}

\begin{abstract}
Photoreactive composite thin layers with tunable wetting properties from superhydrophilic to superhydrophobic nature were prepared. To achieve extreme wetting properties, the adequate surface roughness is a crucial factor, which was achieved by the incorporation of plasmonic $\mathrm{Ag}-\mathrm{TiO}_{2}$ particles, as polymer filler, into the smooth polymer film with adjusted hydrophilicity. The initial copolymer films were synthesized from hydrophilic 2-hydroxyethyl-acrylate (HEA) and hydrophobic perfluorodecyl-acrylate (PFDAc) monomers. In the case of hydrophobic PFDAc, the photocatalyst- roughened thin films displayed superhydrophobic behavior $\left(\gamma_{\mathrm{s}}^{\text {tot }} \sim 2.3 \pm 1.7 \mathrm{~mJ} / \mathrm{m}^{2}, \Theta>150^{\circ}\right)$, while the roughened hydrophilic pHEA layers possessed superhydrophilicity $\left(\gamma_{\mathrm{s}}^{\text {tot }} \sim 72.1 \pm 0.2 \mathrm{~mJ} / \mathrm{m}^{2}, \Theta \sim 0^{\circ}\right)$. The photoactivity of the composites was presented both in solid/ gas $(\mathrm{S} / \mathrm{G})$ and solid/ liquid (S/L) interfaces. According to the light-emitting diode (LED) light photodegradation tests on ethanol (EtOH) as volatile organic compound (VOC) model- molecules at the S/L interface, the superhydrophobic hybrid layer was photooxidized $88.3 \%$ of the initial $\mathrm{EtOH}(0.36 \mathrm{mM})$. At $\mathrm{S} / \mathrm{L}$ interface the photocatalytic efficiency was depended on the polarity of the model pollutant molecules: the photooxidation of hydrophobic SUDAN IV $\left(c_{0}=0.25 \mathrm{mg} / \mathrm{mL}\right) \mathrm{dye}$ reached $80 \%$, while in the case of the hydrophilic Methylene Blue dye $\left(c_{0}=0.002 \mathrm{mg} / \mathrm{mL}\right)$ it was only $17.3 \%$ after $90 \mathrm{~min}$ blue LED light $(\lambda=405 \mathrm{~nm})$ illumination.
\end{abstract}

Keywords: coatings, nanomaterials, polymer composites, surface roughness, adjustable wetting

\section{Introduction}

The superhydrophobic and superhydrophilic behaviors of different surfaces have attracted much attention in industrial fields as well as scientific research $[1,2]$. The superhydrophilic coating material leads to a water contact angle $(\Theta)$ lower than $5^{\circ}$ on solid surface. These materials are already commercially used for antifogging glasses, mirrors and outward walls of buildings. [1,2]. After the discovery of the photo- induced superhydrophilicity of photocatalyst $\left(\mathrm{TiO}_{2}\right)$ layers in 1997 [3], commercially applicable photofunctional materials that have self-cleaning properties were synthetized with the use of pure $\mathrm{TiO}_{2}$ particles and composite thin films [4]. The ultraviolet (UV) light - excitable $\mathrm{TiO}_{2}$ is also well-known as an effective photocatalyst material in the field of environmental remediation or different energy related topics [5, 6]. It is possible to extend the absorption spectrum of the semiconductor photocatalysts by, for example, modifying the catalyst particles with different metallic or non-metallic elements. The synthetized functionalized catalysts can be excited by 
the near-UV or visible (VIS) light due to the absorption of metal nanoparticles at lower energy wavelengths $[5,6]$.

The incorporation of semiconductor photocatalystic particles in an appropriate polymer-based support or binder material is expected to create antimicrobial and self- cleaning properties, which would extend its field of application. The state-of-the-art materials in the area of photocatalyst/ polymer composite layers are the functional surfaces, which are gaining attention for different practical applications, such as selfcleaning superhydrophobic coating [7] and food packing materials with antibacterial behaviors [8]. Polymers are often used as photocatalyst binders due to their low weight, flexibility, low cost and impact resistance. Moreover, the adjustable properties and easy modification of different polymers are also well known from literature [9]. For example, the wetting properties of a polymer surface can be easily adjusted with the monomer composition using hydrophilic and hydrophobic monomers in an adequate ratio [10]. So, if we use polymeric material for the photocatalyst immobilization, a very useful composite surface can be obtained with adjustable properties.

Surfaces with $\Theta$ values higher than $150^{\circ}$ (superhydrophobicity) can exhibit water repellent self-cleaning effects, as found in lotus leaves in nature [11]. For preparing superhydrophobic coating materials, hydrophobic surface functionality as well as surface roughness on two different scales are required: similarly to the surface of the lotus leaf, microstructure is superimposed by nanostructures, and this unique structure, referred to as dual-roughness, can reduce the contact area of water droplets with a surface, resulting in the emergence of water repellent behaviors. The dependence of the $\Theta$ on the surface roughness has been described by Wenzel's or Cassie-Baxter's models [11]. These models can be applied under the assumption that the water droplet is sufficiently larger than the surface roughness. In the past, much effort has been made for preparing superhydrophobic surfaces with high stability [12].

The aforementioned adequate surface roughness necessary for the superhydrophobic nature can be also achieved by the incorporation of photocatalyst particles into a low energy polymer matrix and thus, the study of superhydrophobic photocatalyst/polymer nanocomposite surface is not novel, because many authors reported similar results [13-18]. Nevertheless, these layers were synthetized with difficult preparation techniques such as radio frequency-magnetron sputtering [13], electron beam treatment [14], spin- coating in an inert atmosphere (argon gas) [15], deposition technic in closed chamber and elevated $\left(300^{\circ} \mathrm{C}\right)$ temperature $[16,18]$ or dip- coating process [17] and the obtained coating provides only superhydrophobic properties beside the photoactivity. In our previous work we successfully prepared inorganic/ organic photoreactive composite layers that have also superhydrophobic properties [7]. However, to the best of my knowledge, no studies have been performed yet concerning the adjustable wetting of a photoreactive hybrid layer from superhydrophobic to superhydrophilic properties. In this paper, we present a simple, inexpensive and large scale thin film forming technique for fabricating VIS-light active $\mathrm{Ag}-\mathrm{TiO}_{2}$ plasmonic photocatalyst containing composite surfaces that exhibit adjustable wetting features.

\section{Materials and methods \\ 2.1. Preparation of the $\mathrm{Ag}-\mathrm{TiO}_{2} /$ polyacrylate hybrid layers}

The $\mathrm{Ag}-\mathrm{TiO}_{2}$ plasmonic photocatalyst as polymer filler with $0.5 \mathrm{wt} \%$ silver nanoparticles content was synthesized through the direct functionalization of $\mathrm{TiO}_{2}$ particles. The process is detailed in our previous publications $[5,6]$.

Aqueous fluorochemical cationic dispersion (pPFDAc) fluropolymer - produced by DuPont - (Capstone ST-110, active solid content: $25 \mathrm{wt} \%, \mathrm{pH}=$ 4-6) was used as the hydrophobic binder. The hydrophilic polyacrylate was synthesized by the freeradical UV polymerization of 2-hydroxyethyl acrylate (HEA, purchased from Fluka) in aqueous medium. During the synthesis, 2,2-dimethoxy-2-phenylacetophenone (Irgacure 651, purchased from Aldrich Chemicals) photoinitiator was added in the amount of $0.57 \mathrm{wt} \%$ of the monomer. The mixture was exposed to UV light (light source: Q81 type lamp, Heraeus Gmbh, Hanau, Germany; power: $70 \mathrm{~W} ; \lambda_{\max }=$ $265 \mathrm{~nm}$ ) for 30 minutes. After the polymerization process, the resulting aqueous poly-2-hydroxyethyl acrylate (pHEA) solution (nominal polymer content: $50.0 \mathrm{mg} / \mathrm{mL}$ ) was used in further synthetic steps.

The $5 \times 5 \mathrm{~cm}^{2}$ photoreactive hybrid layers consist of $\mathrm{Ag}-\mathrm{TiO}_{2}$ photocatalysts and polyacrylate binder and $2 \times 2 \mathrm{~cm}^{2}$ smooth polyacrylate layers were prepared by applying the spray-coating technique on glass plate sample holders. The gravimetrically measured 
amounts of material deposited on the glass plates were $5.3 \pm 0.5 \mathrm{mg} / \mathrm{cm}^{2}$. The contents of the photocatalyst in the polyacrylate-based hybrid layers was systematically set to $80.00 \pm 0.11 \mathrm{wt} \%$ to achieve the desired surface roughness, indicated in a previous publication [7]. During the preparation process, aqueous suspensions were prepared with varied pHEA/ pPFDAc content (the pHEA/pPFDAc weight-toweight ratios were $1: 0,8: 2,6: 4,4: 6,2: 8$ and $0: 1$, respectively) and evenly sprayed on 4 and $25 \mathrm{~cm}^{2}$ glass substrates from a distance of $15 \mathrm{~cm}$ using a type R180 Airbrush spray gun at an operating pressure of 3 bar.

\subsection{Methods of characterization}

The surface structure, morphology and roughness of the prepared hybrid thin layers with different pHEA/ pPFDAc contents were examined applying a SEMHitachi S-4700 field emission scanning electron microscope (secondary electron detector, acceleration voltage: 10 or $20 \mathrm{kV}$ ), and a Form Talysurf Series 2 mechanical profilometer (TaylorHobson Ltd., Leicester, Great Britain) at resolutions of $0.25,1 \mu \mathrm{m}$ and $3 \mathrm{~nm}$ in $x, y$ and $z$ directions, respectively. The thickness of the thin composite layers was measured using an Elcometer 224 type digital profile gauge. For optical characterization, diffuse reflectance UV-VIS spectra were recorded via CHEM2000 UV-VIS (USB2000+UV-VIS, Ocean Optics Inc., Dunedin, FL) spectrophotometer, equipped with an integrating sphere [6].

The initial contact angles $(\Theta)$ on $\mathrm{Ag}-\mathrm{TiO}_{2} /$ polymer layers were measured according to the sessile drop technique [7] at $25.0 \pm 0.5^{\circ} \mathrm{C}$ under atmospheric pressure, applying an EasyDrop drop shape analysis system (Krüss GmbH, Hamburg, Germany) equipped with DSA100 software, a Peltier temperature chamber and a steel syringe needle of $0.5 \mathrm{~mm}$ diameter and using distilled water as a test liquid. The initial $\Theta$ were measured after 15 min contact time. During these measurements only the initial $\Theta$ after deposition of water droplet was determined in each case [19]. In the case of the smooth and photocatalyst particles roughened superhydrophobic (r-pPFDAc) and superhydrophilic (r-pHEA) photoreactive thin films the advancing $\left(\Theta_{\text {adv }}\right)$ and receding $\left(\Theta_{\text {rec }}\right)$ contact angles were also measured according to the protocol of Drelich [20]. According to the theory of Drelich and Chibowski [21] the obtained $\Theta_{\mathrm{adv}}$ and receding $\Theta \mathrm{r}_{\mathrm{ec}}$ are also suitable for the estimation of the total apparent surface free energy $\left(\gamma_{\mathrm{s}}^{\text {tot }}\right)$ of the layer, knowing the surface tension of the probe liquid, $\left(\gamma_{1}\right.$, $72.1 \mathrm{mN} / \mathrm{m}$ in the case of distilled water at $25^{\circ} \mathrm{C}$ ) and its contact angle hysteresis, which is defined as the difference between the $\Theta_{\text {adv }}$ and $\Theta_{\text {rec }}$, as shown by Equation (1):

$\gamma_{\mathrm{s}}^{\text {tot }}=\frac{\gamma_{1}\left(1+\cos \Theta_{\mathrm{adv}}\right)^{2}}{2+\cos \Theta_{\mathrm{rec}}+\cos \Theta_{\mathrm{adv}}}$

The apparent dynamic $\Theta$ of the hybrid layers were also determined by the Wilhelmy plate method. A digital surface tensiometer was applied (Force tensiometer - K100, Krüss GmbH, Hamburg, Germany). The $19.5 \times 12.8 \times 0.3 \mathrm{~cm}^{3}$ steel plates coated with hybrid layers $\left(5.3 \pm 0.5 \mathrm{mg} / \mathrm{cm}^{2}\right.$ on both sides $)$ were immersed into distilled water with a rate of $6 \mathrm{~mm} / \mathrm{min}$, while the changes in apparent $\Theta_{\text {adv }}$ and $\Theta_{\text {rec }}$ were calculated online by LabDesk 3.2 Software. The measurements were run at $25.0 \pm 0.5^{\circ} \mathrm{C}$.

The photocatalytic properties of the $\mathrm{Ag}-\mathrm{TiO}_{2} /$ polyacrylate hybrid layers were studied during ethanol degradation tests under blue LED light (General Electric's, Hungary, $\lambda=405 \mathrm{~nm}$ ) illumination at $\mathrm{S} / \mathrm{G}$ interface. To quantify the degradation of EtOH test molecules, gas chromatographic (GC) measurements were carried out (Shimadzu GC-14B, Kyoto, Japan). During the measurements, $5 \mu \mathrm{L}$ of EtOH (abs., Molar Chemicals, Hungary) was injected into the reaction chamber containing $25 \mathrm{~cm}^{2}\left(25.0 \pm 0.5^{\circ} \mathrm{C}\right)$ hybrid layers, fixed at $5 \mathrm{~cm}$ distance from the light source. The $c / c_{0}$ values calculated from peak areas were determined as the function of illumination time, where $c$ is the concentration of EtOH at time $(t)$ and $c_{0}$ is the initial concentration $\left(c_{0}=0.36 \mathrm{mM}\right)$. The apparent reaction rate constants $\left(k^{\prime}\left[\mathrm{min}^{-1}\right]\right.$ were calculated for first-order decay as shown by Equation (2) [7]:

$\ln \frac{c}{c_{0}}=-k^{\prime} t$

The photocatalytic activity of the hybrid layers was also measured at $\mathrm{S} / \mathrm{L}$ interface. During these measurements hydrophilic Methylene Blue (Reanal, Hungary) in aqueous medium (distilled water) and hydrophobic SUDAN IV (Reanal, Hungary) dye in organic medium (ethanol with $5 \mathrm{v} / \mathrm{v} \%$ water content) were chosen as model pollutants to evaluate the photocatalytic activity of the hybrid layers. The photocatalytic activity, i.e. the decreasing concentration of the dyes were measured with a spectrophotometer and the tests were carried out directly in a quartz cuvette 
containing the superhydrophobic photoreactive thin film $\left(3 \times 0.9 \mathrm{~cm}^{2}\right)$ and the dye solutions $(3 \mathrm{~mL})$. The absorbance spectra were taken after $0,10,20,30,40$, $50,60,75$ and 90 min of LED light illumination. The concentration of the dyes were $0.25 \mathrm{mg} / \mathrm{mL}$ and $0.002 \mathrm{mg} / \mathrm{mL}$ in the case of hydrophobic SUDAN IV and hydrophilic Methylene Blue, respectively.

\section{Results and discussion}

In our previous work, UV- excitable $\mathrm{ZnO}$ containing photocatalyst particles with primer mean particle diameter of $20 \mu \mathrm{m}$ were immobilized in a low energy fluoropolymer binder to obtain bifunctional thin layers: besides the photocatalytic activity, lotus leaf-like wettability was achieved due to the incorporated photocatalyst particles enhanced surface roughness [7]. To achieve such wetting properties, the amount of the immobilized photocatalyst was proven to be at least $80 \mathrm{wt} . \%$. At this composition the measured surface roughness value of the hybrid layer was $15.2 \mu \mathrm{m}$ and this rough, thin film exhibits water repellent superhydropobic properties. In this work, the wetting properties of layers with adequate surface roughness were modified via the systematic adjustment of hydrophilic pHEA and hydrophobic pPFDAc besides keeping the previously determined photocatalyst-to-polymer ratio $(8: 2)$ to achieve a wide range of wettability, from superhidrophylicity to superhydrophobicity.

\subsection{Wetting properties of thin films and the determined surface energy values}

The wetting properties of the smooth polymer thin films with varied composition and the corresponding roughened hybrid layers was determined by static/ initial $\Theta$ measurements. The $\Theta$ values were measured after 15 min of contact time. Figure 1 shows the measured initial $\Theta$ as a function of polymer composition. The increasing hydrophobic fluoropolymer content (from left to right) was increased the measured $\Theta$ values, i.e. decreased the wettability of the polymer layer. The initial pHEA layer has a relative low $\Theta$ value $\left(27.4^{\circ}\right)$ indicating the hydrophilic nature of the polymer. However, at $20 \mathrm{wt} \%$ of fluoropolymer-content in the binder the measured $\Theta$ value was achieved the $60^{\circ}$ and gradually increased to $105^{\circ}$ with the increasing pPFDAc content. Results of static contact angle measurements were also confirmed, that the composite films roughened by $\mathrm{Ag}-\mathrm{TiO}_{2}$ photocatalyst particles showed highlighted wetting properties

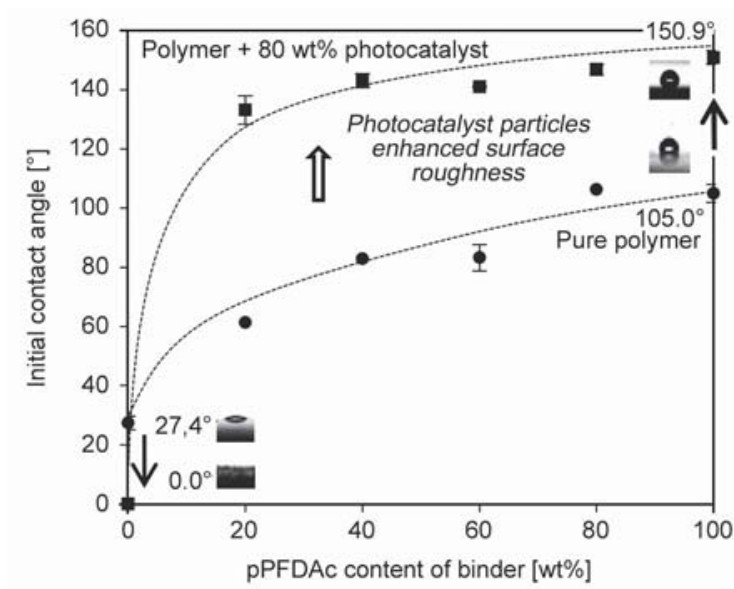

Figure 1. Measured apparent static initial water contact angles on pure polyacrylate thin films and $\mathrm{Ag}-\mathrm{TiO}_{2}$ photocatalyst particle roughened polyacrylate hybrid layers as a function of the hydrophobic pPFDAc content of the copolymer binder $(T=$ $\left.25 \pm 0.5^{\circ} \mathrm{C}\right)$.

compared to the pure polymer thin layers, and even superhydrophilic characteristic $\left(\Theta=0^{\circ}\right)$ could be achieved at $100 \mathrm{wt} \%$ pHEA content in the copolymer. The results showed that the measured $\Theta$ values of polymer films with flat surfaces were varied between 27.4 and $105.0^{\circ}$, however, the wetting properties of the photocatalyst- roughened surfaces can be adjusted from superhydrophilic (pHEA; $\Theta=\sim 0^{\circ}$ ) to superhydrophobic (pPFDAc; $\Theta=150.9^{\circ}$ ). So, the initial wetting character of smooth thin layers was highlighted by the increased surface roughness caused by the photocatalyst incorporation into the polymer matrix.

The wetting properties of the smooth and $\mathrm{Ag}-\mathrm{TiO}_{2}$ particles roughened (r-pPFDAc) and (r-pHEA) composite layers were also studied by dynamic advancing $\left(\Theta_{\text {adv }}\right)$ and receding $\left(\Theta_{\text {rec }}\right)$ contact angles measurements (Figure 2a). These were carried out according to the protocol of Drelich [20]. In the case of superhydrophobic r-pPFDAc layer, increasing the volume of the water drop leads to reduction of the $\Theta_{\text {adv }}$ from $\Theta_{7.5 \mu \mathrm{L}}=165.2^{\circ}$ to $\Theta_{23.0 \mu \mathrm{L}}=150.6^{\circ}$. Note, that this latter value is very close to the initial $\Theta$ value $\left(150.9^{\circ}\right)$, which was measured after 15 min contact time, indicating the steady state condition of the initial/static water contact angle (Figure 1.). The $\Theta_{\text {rec }}$ were gradually decreasing as the volume of the drop was decreasing and the final value was much lower $\left(125.0^{\circ}\right)$ than the static contact angle $\left(150.9^{\circ}\right)$. This is maybe due to the presence of surface hydrophilic photocatalyst particles, which caused that it is difficult to remove liquid from surfaces during the measurements 

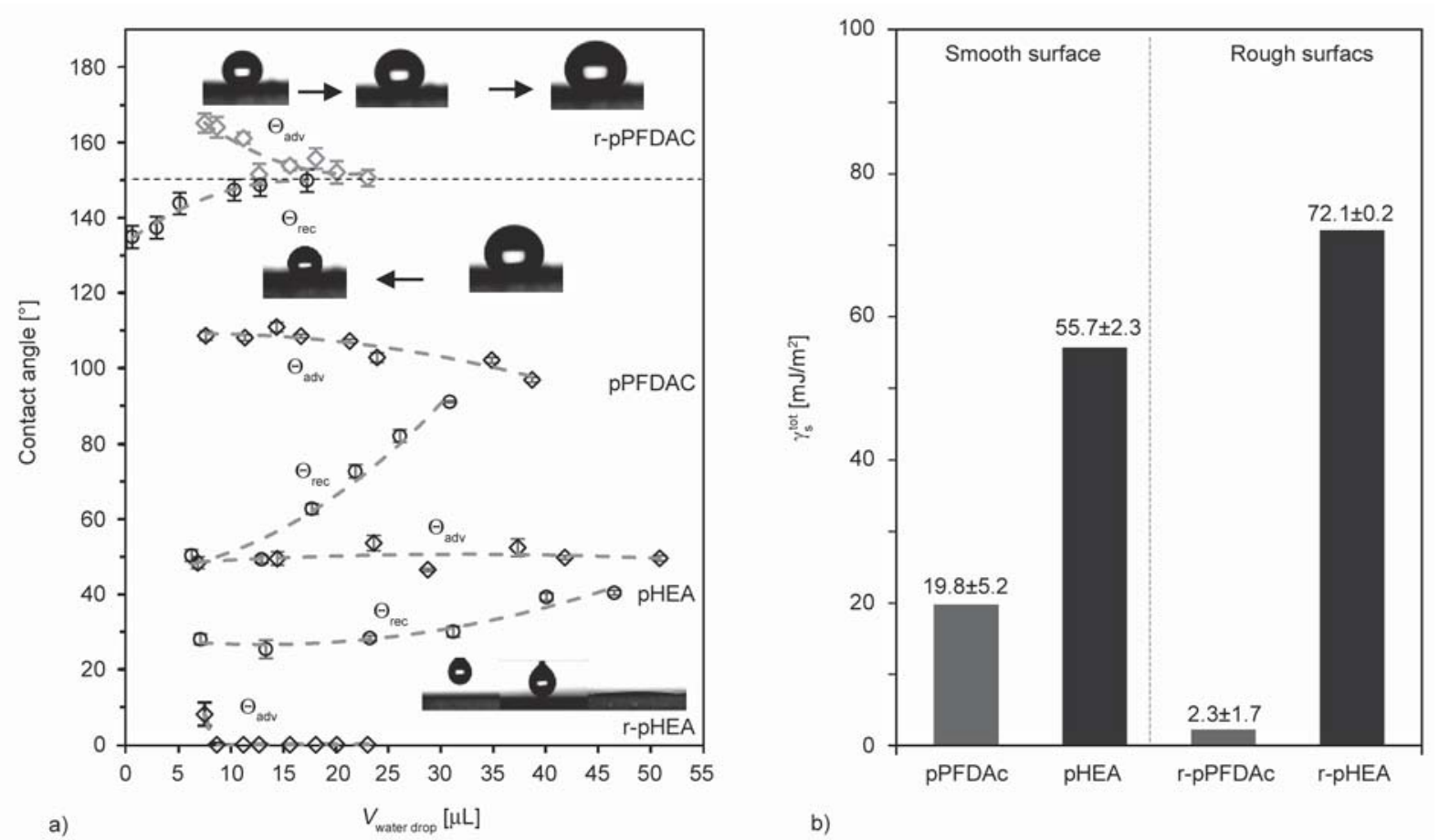

Figure 2. Evolution of advancing and receding contact angles on smooth and roughened (r-) pPFDAc and pHEA hybrid layers (a), as well as the determined total apparent surface free energy $\left(\gamma_{\mathrm{s}}^{\mathrm{tot}}\right)$ values of initial (smooth) and roughened polyacrylate thin layers (b).

of $\Theta_{\text {rec. }}$ In the case of the smooth hydrophobic pPFDAc polymer layer the obtained $\Theta_{\text {adv }}$ and $\Theta_{\text {rec }}$ values were $\sim 110$ and $\sim 45^{\circ}$, respectively, while in the case of hydrophilic smoot pHEA film these values were $\sim 35$ and $\sim 24^{\circ}$, respectively. As regards the superhydrophilic r-pHEA hybrid layer, it was impossible to adequately measure the dynamic contact angle because even the smallest drop tended to spread very rapidly. A gradual increase of the drop volume led to further spreading and the contact angles were far too small to be measured accurately. This is also indicating the very well wetting, superhydrophilic nature of the film.

According to the theory of Chibowski and coworkers $[21,22]$ the obtained $\Theta_{\text {adv }}$ and $\Theta_{\text {rec }}$ are also suitable for the estimation of the total apparent surface free energy ( $\gamma$ stot) of the layer (see Equation (1)). The determined $\gamma$ stot values of the pure hydrophobic pPFDAc and hydrophilic pHEA thin layers were $19.8 \pm 5.2$ and $55.7 \pm 2.3 \mathrm{~mJ} / \mathrm{m}^{2}$ respectively. This result agrees with our previous measurements and the previously-reported surface energy values of the low-energy flat Teflon-like surfaces [7]. The roughening-induced changes in $\gamma_{\mathrm{s}}^{\text {tot }}$ values are represented in Figure 2b. The enhanced surface roughness of a hybrid layer containing $80 \mathrm{wt} \% \mathrm{Ag}-\mathrm{TiO}_{2}$ caused a drastic change in the measured $\gamma$ stot value: in the case of high energy (hydrophilic) pHEA polymer binder the measured value of roughened surface was increased up to $72.1 \pm 0.2 \mathrm{~mJ} / \mathrm{m}^{2}$, while in the case of low energy pPFDAc polymer the value was decreased to $2.3 \pm 1.7 \mathrm{~mJ} / \mathrm{m}^{2}$. In other words, the hydrophilic/hydrophobic nature of the initial smooth surface was enhanced with the surface roughness, the hydrophilic surface becomes more hydrophilic or superhydrophilic, while the low energy hydrophobic hybrid layer showed superhydrophobic properties after the roughening process.

Dynamic contact angles were also determined applying the Wilhelmy plate method [23]. The plots inFigure 3 represent online contact angle data calculated during the measurements. It should be noted, however, that dynamic contact angle measurements have been made for a wide variety of different liquids and surfaces [24, 25], the dynamic wetting characterization of superhydrophobic surfaces is difficult and can easily misinterpreted, because a reliable dynamic measurement requires homogenous sample, relative smooth surface and symmetric shape [26]. In this case a good run will show the slope of the advancing and receding portions of the plot, which is constant in the mean and equal for both stages of the measurement: the two lines must be parallel, a very 

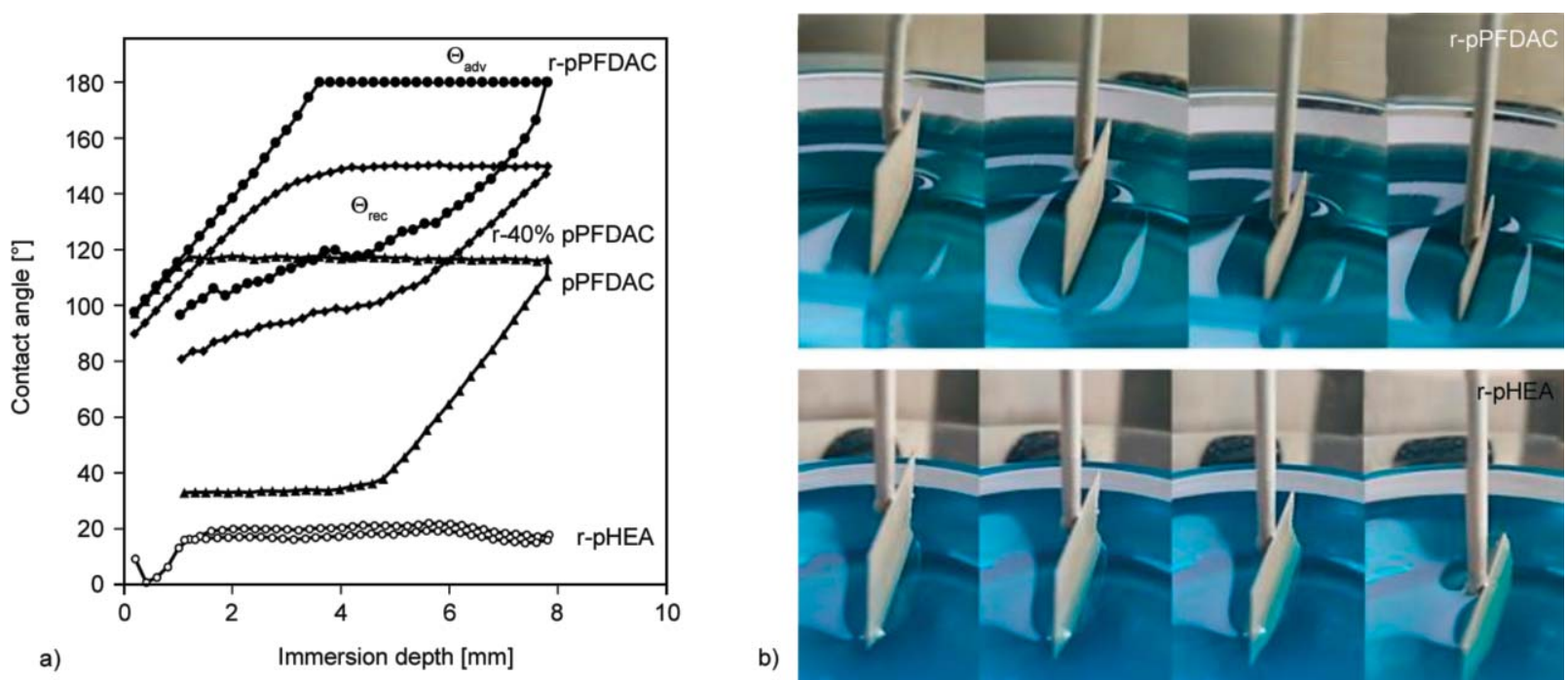

b)

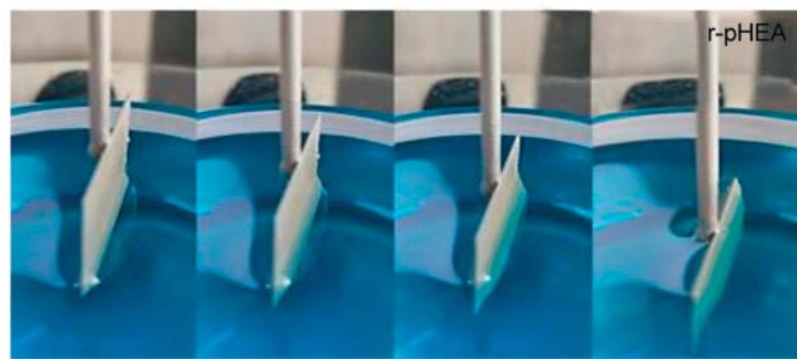

Figure 3. Directly measured apparent dynamic water contact angles on polyacrylate-based thin films (the r-stands for roughened) as a function of the immersion depth of the coated Wilhelmy-plate $\left(T=25 \pm 0.5^{\circ} \mathrm{C}\right)$ (a), as well as the photos of the r-pPFDAc and r-pHEA coated plates during the immersion process in distilled water (coloured with methylene blue for better imagine) determined average dynamic contact angles on polyacrylate-based thin films as a function of the binder composition (b).

simple trick which may also work as an internal quality check of the run [29].

In the case of flat fluoropolymer (pPFDAc) coating this tendency can be observed $\left(\Theta_{\mathrm{adv}}=\sim 118^{\circ} ; \Theta_{\mathrm{rec}}=\right.$ $\sim 33^{\circ}$ ), however, the roughened composite layer (r-pPFDAc) has shown disturbed and non-parallel lines. This is obviously due to the rough and heterogeneous surface and the obtained $180^{\circ}$ advancing contact angle is also should be handle with care because there is no justification for experimental contact angle of $180^{\circ}$ unless liquid if floating above the surface like in the case water droplet placed on a hot plate. However, the non-wetting nature of the r-pPFDAc layer is clearly indicated the inserted photo in Figure 3. The immersion of plate with superhydrophobic coating resulted in dented water surface during the measurement, while in the case of superhydrophilic coating (r-pHEA) the meniscus climbed up due to the capillary forces $\left(\Theta_{\mathrm{adv}}=\sim 20^{\circ} ; \Theta_{\mathrm{rec}}=\sim 17^{\circ}\right)$.

The results of the dynamic contact angle measurements also prove the expanded wetting range of the polyacrylate-based layers and it can be seen that the wetting properties of the composite layers could be tailored by the hydrophilicity of the polymer matrix.

\subsection{Surface morphology and porosity of the composites}

The dependence of surface roughness on the wetting properties (i.e. the measured liquid contact angle) has been described by Wenzel's or Cassie-Baxter's models [11]. A low surface free energy flat surface must be roughened to get superhydrophobic surfaces with high $\left(\Theta>150^{\circ}\right)$ water contact angle [7]. The captured SEM images (Figure $4 \mathrm{a}-4 \mathrm{c}$ ) show the relative smooth surface of the initial, $\mathrm{Ag}-\mathrm{TiO}_{2}$ photocatalyst-free pure fluoropolymer layer. The prospected flat surface was also evidenced by mechanical profilometric measurements (Figure 5). The measured $R_{\mathrm{q}}$ values of the initial pHEA and pPFDAc polymer surfaces were $2.1 \pm 0.8$ and $3.7 \pm 0.9 \mu \mathrm{m}$, respectively. However, in the case of the photocatalyst nanoparticles roughened hybrid layers quasi-spherical microscale-aggregates of the primer photocatalyst particles can be observed on the composite surface with diameters ranging from 5 to $40 \mu \mathrm{m}$ (Figure $4 \mathrm{~d}-4 \mathrm{f}$ ). Accordingly, the determined $R_{\mathrm{q}}$ values of the roughened r-pHEA and r-pPFDAc hybrid surfaces were $11.7 \pm 2.2$ and $15.2 \pm 1.2 \mu \mathrm{m}$, respectively. Moreover, according to the SEM pictures, at increased magnifications, nanostructures could be identified superposed on the microscale structure.

This is due to the nanoparticles aggregation during the composite film forming process: the initial Ag$\mathrm{TiO}_{2}$ photocatalyst particles with $\sim 50 \mathrm{~nm}$ primary (nominal) particle size were aggregated into 5-40 $\mu \mathrm{m}$ microparticles. This structure ensures high porosity and accessibility which is advantageous for photocatalytic process. However, it is also worth to note, that the reported penetration depth of UV light ( $254 \mathrm{~nm}$ ) into porous $\mathrm{TiO}_{2}$ film is just a few micrometer [27], 

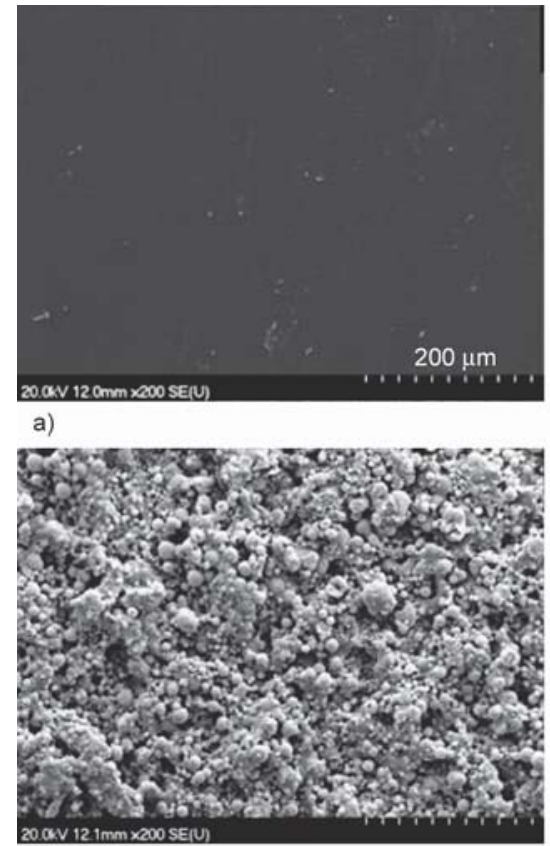

d)

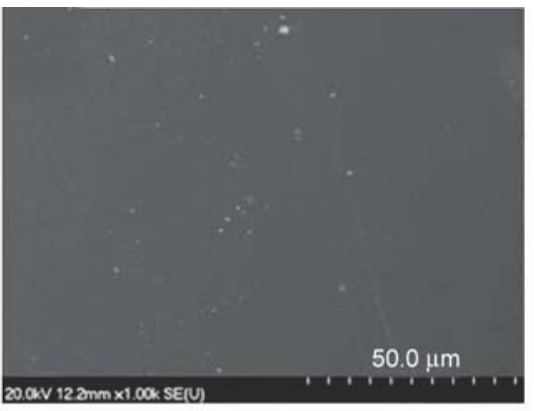

b)

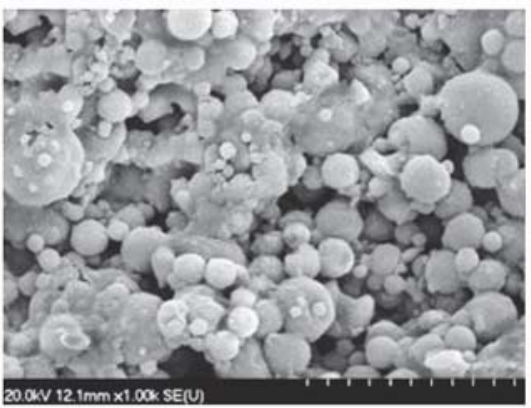

e)

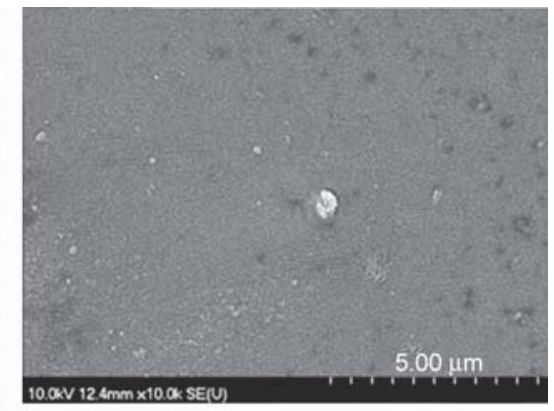

c)

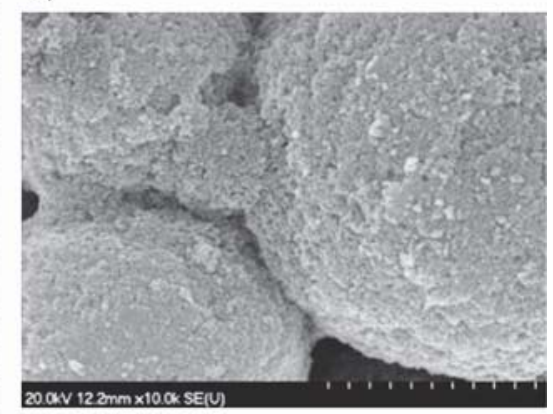

f)

Figure 4. SEM images of the prepared smooth pPFDAc polymer layer $(\mathrm{a}-\mathrm{c})$ and the $80 \mathrm{wt} \% \mathrm{Ag}-\mathrm{TiO}_{2}$ containing pPFDAc (df) thin layers with different magnifications: $200 \times(a, d), 1000 \times(b, e), 10000 \times(c, f)$.

thus photooxidation actually take place only at surface of the $100 \mu \mathrm{m}$ porous film. Due to this phenomenon the photoreactive hybrid layers were shown rough surface both in micro- and nanoscale. The average thickness of the composite layers was measured by Elcometer 224 type digital profile gauge and found to be $91.3 \pm 10.4 \mu \mathrm{m}$. Beside this measured layer thickness value, the calculated film thickness was also determined from the specific surface amount of hybrid layers $\left(5.3 \pm 0.5 \mathrm{mg} / \mathrm{cm}^{2}\right)$ and the previously determined density of the components $\left(\rho_{\mathrm{Ag}^{-\mathrm{TiO}_{2}}}\right.$ : $4.32 \mathrm{~g} / \mathrm{cm}^{3}$; polymer: $1.10 \mathrm{~g} / \mathrm{cm}^{3}$ ). According to the calculation, the average layer thickness of the hybrid

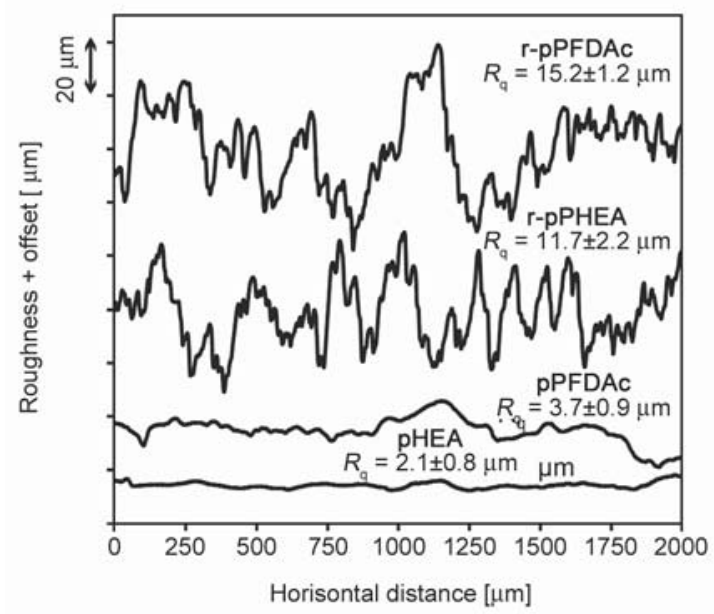

Figure 5. Measured profilometer data for the prepared pure polymer thin layers and the corresponding photocatalyst roughened photoreactive hybrid films. layers was $19.3 \pm 3.2 \mu \mathrm{m}$. The difference between the measured and calculated values are referred to the film porosity [7]. The results revealed that the calculated average porosity value was $78.1 \%$.

\subsection{Characterization of optical and photocatalytic properties of thin films}

The optical parameters of polyacrylate binder and polyarcrylate $/ \mathrm{Ag}-\mathrm{TiO}_{2}$ hybrid layers were studied and compared applying UV-VIS diffuse reflectance spectroscopy (Figure 6). The absorption spectrum of the initial plasmonic $\mathrm{Ag}-\mathrm{TiO}_{2}$ photocatalyst sample was also presented for reference. As demonstrated in our previous papers, the presence of silver nanodots on the surface of semiconductor $\mathrm{TiO}_{2}$ photocatalysts, resulted in a plasmonic peak in the visiblelight region with a wavelength maximum of $450 \mathrm{~nm}$ $[5,6]$. It was also proven that these plasmonic photocatalysts presented increased photocatalytic activity under blue (405 nm) LED light illumination compared to the initial $\mathrm{P} 25 \mathrm{TiO}_{2}[5,6]$. In this noble metal-semiconductor composite photocatalyst, visible light is harvested due to the surface plasmon resonance of the AgNPs, while the metal-semiconductor interface separates the generated electrons and holes. The emission spectra of the used LED were also presented in Figure 6 (grey dashed line). The plasmonic peak and the well- known UV- light absorbance edge of $\mathrm{TiO}_{2}$ were also presented in the 


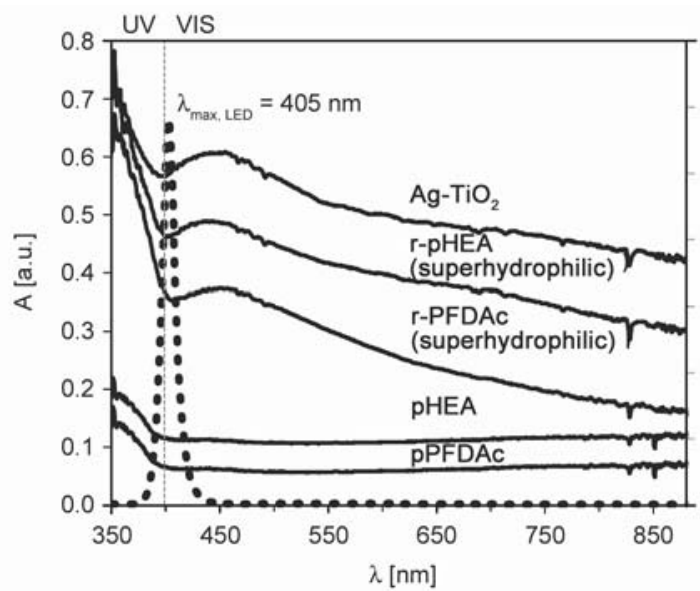

Figure 6. Diffuse reflectance spectra of the prepared hybrid thin layers and the initial plasmonic $\mathrm{Ag}-\mathrm{TiO}_{2}$ photocatalyst, as well as the emission spectrum of the LED lamp used in photodegradation tests (dashed line).

case of the roughened hybrid films containing $80 \mathrm{wt} \%$ plasmonic photocatalyst particles.

However, the absorbance spectra of the initial hydrophilic pHEA and hydrophobic pPFDAc binders indicate the transparent nature of the polymer thin films. So, the transparent polymer binder material with adjustable wetting properties serve as support material for the attached plasmonic photocatalyst nanoparticles and ensure high photocatalyst dispersibility and mechanical stability. The improved mechanical stability of the polymer binder based

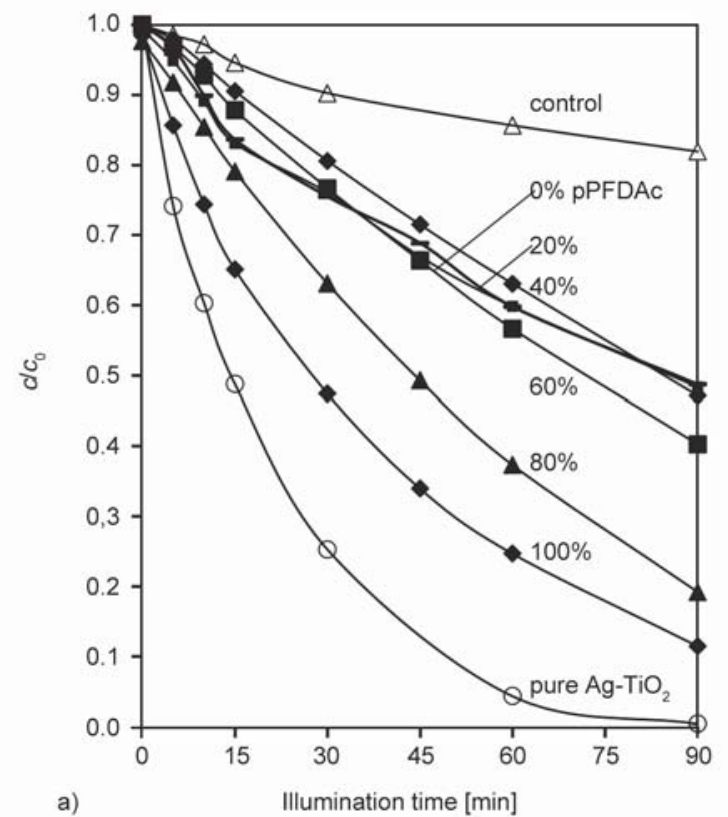

photoreactive hybrid thin films was reported elsewhere using the adhesive tape test method [5].

After the wetting, morphological and optical properties of the hybrid layers the photocatalytic behavior was also measured. The photocatalytic properties of the composite thin films were studied both at $\mathrm{S} / \mathrm{G}$ and $\mathrm{S} / \mathrm{L}$ interface, as well. As a result of $90 \mathrm{~min}$ of blue LED light (Figure 6) illumination of the samples $\left(\lambda_{\max }=405 \mathrm{~nm}\right)$, the layers consist of pure photocatalyst decomposed approximately the whole amount of the model compound at the solid-gas interface (the initial $\mathrm{EtOH}$ concentration was $0.36 \mathrm{mM}$ ), while the immobilized catalyst showed moderate reaction rates (Figure 7a), furthermore, an unambiguous growth-tendency was observed considering the decomposition rate constants $\left(k^{\prime}\right)$ as the fluoropolymer content increased (Figure 7b). The data points labelled as 'control' in Figure 7a represent the photodegradation of $\mathrm{EtOH}$ in the absence of photoreactive thin layers (glass slide sample holder). It can be seen, that the degree of photolysis was ca. $12 \%$, however, in the presence of photocatalysts, the films exhibited much higher photooxidation rate. In the case of the r-pPFDAc layer the amount of decomposed EtOH $(88.3 \%$ decrease in the $0.36 \mathrm{mM}$ initial concentration) was comparable with the efficiency experienced in the case of pure $\mathrm{Ag}-\mathrm{TiO}_{2}$ films (99.4\%). So the photocatalytic activity of this layers were

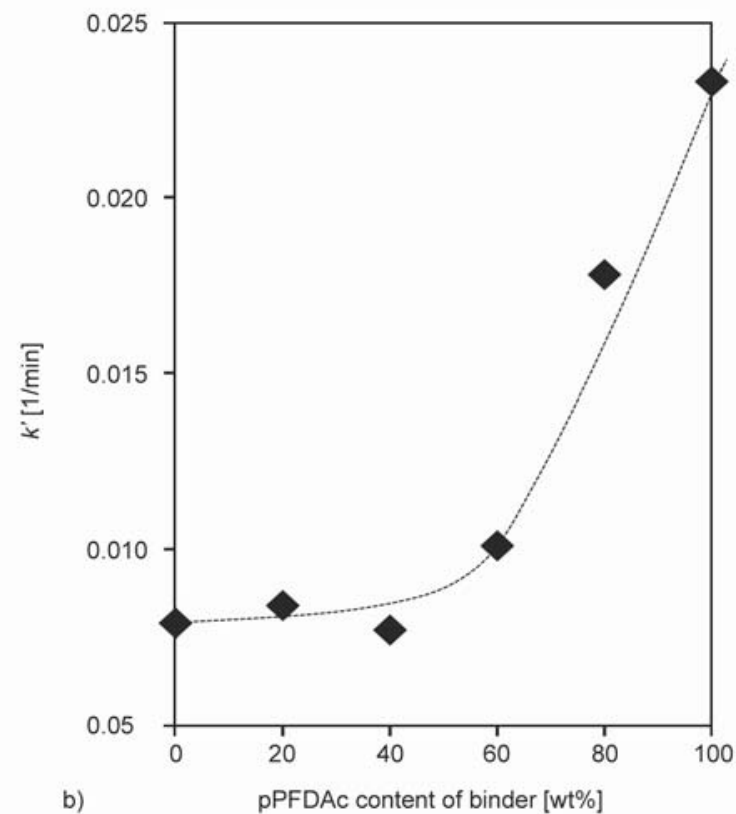

Figure 7. Characterization of EtOH decomposition on $\mathrm{Ag}-\mathrm{TiO}_{2} /$ polyarcrylate hybrid layers under LED light illumination $(\lambda=405 \mathrm{~nm})$ as the function of illumination time (the legends refer to the composition of the polymer binder) (a) and the determined apparent photocatalytic oxidation rate constants for first-order decay as a function of pPDAc content of the binder $\left(\ln \left(c / c_{0}\right)=-k^{\prime} t\right)(b)$. 
evidenced at $\mathrm{S} / \mathrm{G}$ interface using ethanol test molecules as model VOC.

Next the photoactivity of the hybrid layers was also presented at the $\mathrm{S} / \mathrm{L}$ interface. The superhydrophilic nature of an irradiated semiconductor surface is not unusual, because under UV- irradiation $\mathrm{TiO}_{2}$ acquires superhydrophilic properties [1]. However, the photocatalytic activity of a photoreactive and even superhydrophobic coating is not yet reported in the literature. Thus, the photocatalytic behavior of the superhydrophobic layer was also demonstrated and studied in the case of hydrophilic and hydrophobic model pollutants (Figure 8). According to the results the hydrophilic aqueous Methylene Blue solution did not wet the surface of the hybrid film (see inserted photo inFigure $8 \mathrm{~b}$ with blue aqueous liquid drop) and therefor the ratio of the photodegraded Methylene Blue dye $\left(c_{0}=0.002 \mathrm{mg} / \mathrm{mL}\right)$ was only $17.3 \%$ after 90 min blue LED light $(\lambda=405 \mathrm{~nm})$ irradiation. However, the photooxidation of hydrophobic SUDAN IV solutions ( $\left.c_{0}=0.25 \mathrm{mg} / \mathrm{mL}\right)$ almost reached the $80 \%$. This is because the adequate wetting of the superhydrophobic film surface by hydrophobic medium (see the red dye stain in Figure 8b), which means high contact area between the surface photocatalyst particles and the model pollutants molecules. When

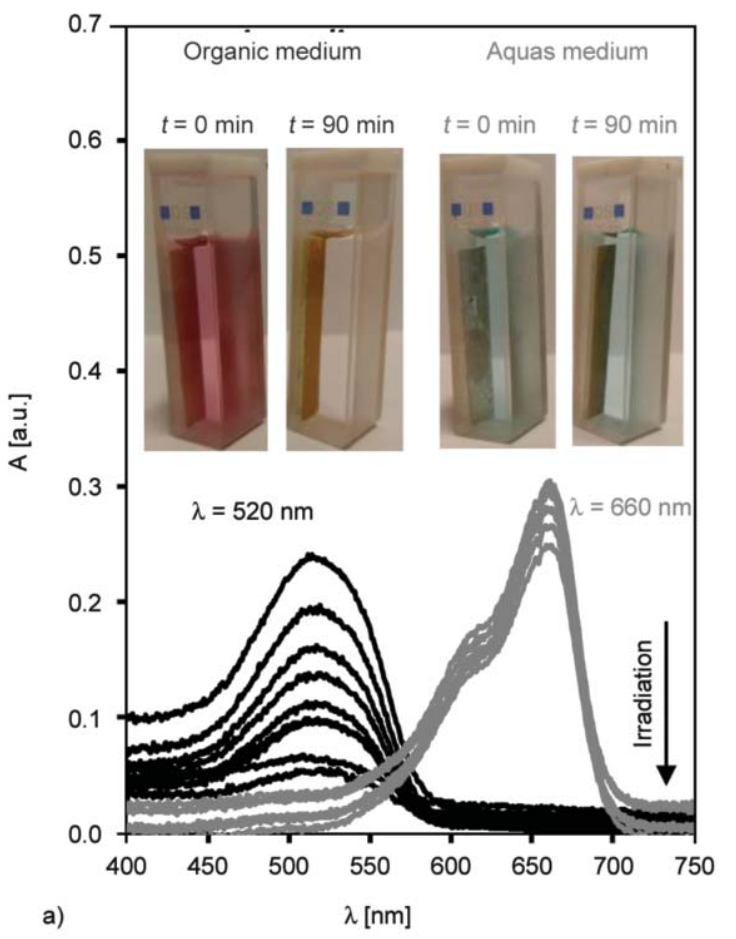

the hydrophobic surface, contacted earlier with air, is immersed into water then air can stay attached to the surface [28]. This air film also can be seen on the surface of the film in aqueous medium at $t=0 \mathrm{~min}$ irradiation (Figure 8a).

Finally, it is also should be noted that self-photodegradation of the polymer binder could cause problem during long term irradiation time as reported in our previous paper [5]. The reason for this is that the light induced reactive oxygen species also can destroy the organic polymer matrix. However, we have reported that this undesired photodedradation can be avoided by the application of appropriate composite catalyst [29]. Hydroxyapatite ( $\mathrm{HAp}) / \mathrm{TiO}_{2}$ composite catalyst was synthetized by co-precipitation method, where the HAp microlamellae serve as support material for the surface attached $\mathrm{TiO}_{2}$ particles. Thus, HAp serves as a spacer to prevent direct contact polymer and $\mathrm{TiO}_{2}$, so the photodegradation of polymer was significantly reduced [29].

\section{Conclusions}

Photoreactive hybrid layers with adjustable wetting properties were prepared with micro- and nanosized dual-scale surface roughness. The films with composition- dependent wetting properties consist of

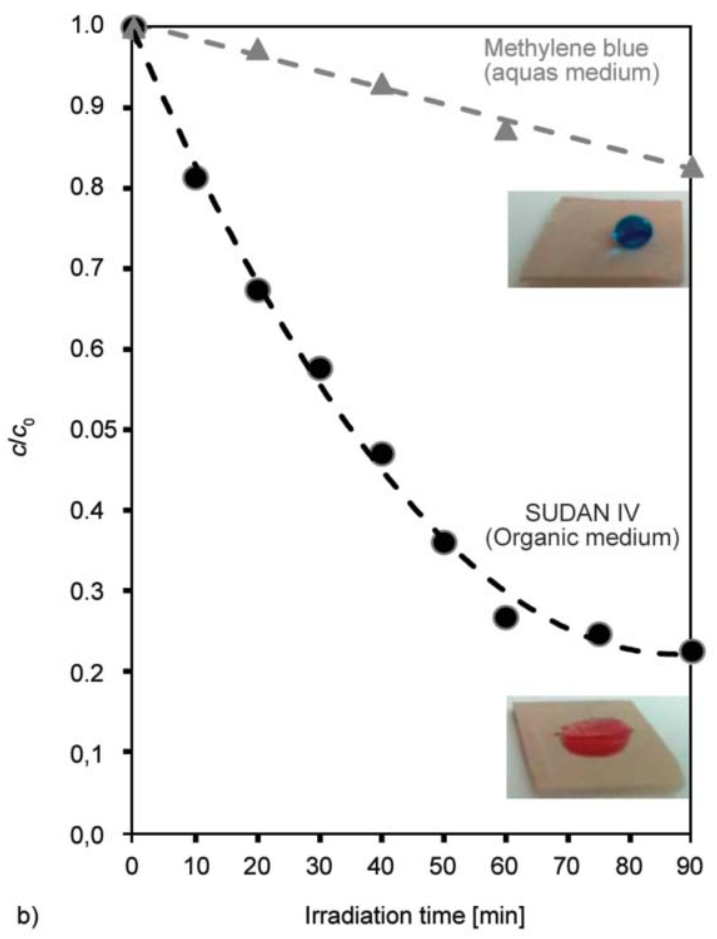

Figure 8. The effect of LED light $(\lambda=405 \mathrm{~nm})$ irradiation on the absorbance spectra of the hydrophobic SUDAN IV and aqueous Methylene Blue solutions (a) and the corresponding normalized concentration changes (b) of dyes. The inserted images show the photos of dye solutions before and after irradiation (a) and the aqueous and hydrophobic liquid drops on the surface of the superhydrophobic hybrid film (b). 
plasmonic $\mathrm{Ag}-\mathrm{TiO}_{2}$ photocatalyst particles (80 wt $\%$ ) and transparent copolymer binder (20 wt $\%$ ) with tailored hydrophilicity. Thus, the initial wetting properties of high energy pHEA $\left(\Theta=27.4^{\circ}, \gamma_{\mathrm{s}}^{\text {tot }}=\right.$ $\left.55.7 \pm 2.3 \mathrm{~mJ} / \mathrm{m}^{2}\right)$ and low energy pPFDAc $(\Theta=$ $105.0^{\circ}, \gamma=\gamma_{\mathrm{s}}^{\text {tot }}=19 . \pm 5.2 \mathrm{~mJ} / \mathrm{m}^{2}$ ) flat polymer films were increased with the photocatalyst enhanced surface roughness. In the case of roughened pHEA and pPFDAc layers superhydrophilic $\left(\Theta=0^{\circ}, \gamma \geq \gamma_{\mathrm{s}}^{\text {tot }}=\right.$ $\left.72.1 \pm 02 \mathrm{~mJ} / \mathrm{m}^{2}\right)$ and superhydrophobic $\left(\Theta=150.9^{\circ}\right.$, $\left.\gamma_{\mathrm{s}}^{\text {tot }}=2.3 \pm 1.7 \mathrm{~mJ} / \mathrm{m}^{2}\right)$ characters were experienced, respectively. The photocatalytic properties of the thin layers were first measured at $\mathrm{S} / \mathrm{G}$ interface using ethanol as model VOC test molecules and the photodegraded percentage amount of the initial $0.36 \mathrm{mM}$ EtOH varied between 51.3 and $88.3 \%$. This is due to the porous structure of the thin films with high inner surfaces. However, at $\mathrm{S} / \mathrm{L}$ interface the photocatalytic efficiency was depend on the polarity of the used model pollutants: the superhydrophobic rough thin film was photodegraded only the hydrophobic SUDAN IV dye solution. The advantage of this current surface compared to previous photoreactive composite coatings is the monomer composition dependent wetting properties. These dual photocatalytic and adjustable wetting properties of the prepared composite layers could be interesting at the area of water treatment, where the polarity of the pollutants varies on a wide scale.

\section{Acknowledgements}

The authors are very thankful for the financial support from the Hungarian Scientific Research Fund (OTKA) K 116323, PD 116224 and the project named GINOP-2.3.2-15-201600013. D. Sebők gratefully acknowledges the financial support of J. Bolyai research fellowship.This paper was supported by the UNKP-17-4 New National Excellence Program of the Ministry of Human Capacities (L. Janovák.).

\section{References}

[1] Fujishima A., Rao T. N., Tryk D. A.: Titanium dioxide photocatalysis. Journal of Photochemistry and Photobiology C: Photochemistry Reviews, 1, 1-21 (2000). https://doi.org/10.1016/S1389-5567(00)00002-2

[2] Ma X., Shen B., Zhang L., Liu Y., Zhai W., Zheng W.: Porous superhydrophobic polymer/carbon composites for lightweight and self-cleaning EMI shielding application. Composites Science and Technology, 158, 8693 (2012).

https://doi.org/10.1016/j.compscitech.2018.02.006
[3] Wang R., Hashimoto K., Fujishima A., Chikuni M., Kojima E., Kitamura A., Shimohigoshi M., Watanabe T.: Light-induced amphiphilic surfaces. Nature, 388, 431432 (1997).

https://doi.org/10.1038/41233

[4] Chen Z. M., Pan S. J., Yin H. J., Zhang L. L., Ou E. C., Xiong Y. Q., Xu W. J.: Facile synthesis of superhydrophobic $\mathrm{TiO}_{2}$ /polystyrene core-shell microspheres. Express Polymer Letters, 5, 38-46 (2011). https://doi.org/10.3144/expresspolymlett.2011.5

[5] Veres Á., Ménesi J., Juhász Á., Berkesi O., Ábrahám N., Bohus G., Oszkó A., Pótári G., Buzás N., Janovák L., Dékány I.: Photocatalytic performance of silver-modified $\mathrm{TiO}_{2}$ embedded in poly(ethyl-acrylate-co-methyl metacrylate) matrix. Colloid Polymer Science, 292, 207-217 (2014).

https://doi.org/10.1007/s00396-013-3063-1

[6] Veres Á., Rica T., Janovák L., Dömök M., Buzás N., Zöllmer V., Seemann T., Richardt A., Dékány I.: Silver and gold modified plasmonic $\mathrm{TiO}_{2}$ hybrid films for photocatalytic decomposition of ethanol under visible light. Catalysis Today, 181, 156-162 (2012).

https://doi.org/10.1016/j.cattod.2011.05.028

[7] Deák Á., Janovák L., Csapó E., Ungor D., Pálinkó I., Puskás S., Ördög T., Ricza T., Dékány I.: Layered double oxide (LDO) particle containing photoreactive hybrid layers with tunable superhydrophobic and photocatalytic properties. Applied Surface Science, 389, 294 302 (2016).

https://doi.org/10.1016/j.apsusc.2016.07.127

[8] Zhang X., Xiao G., Wang Y., Zhao Y., Su H., Tan T.: Preparation of chitosan- $\mathrm{TiO}_{2}$ composite film with efficient antimicrobial activities under visible light for food packaging applications. Carbohydrate Polymers, 169, 101-107 (2017).

https://doi.org/10.1016/j.carbpol.2017.03.073

[9] Janovák L., Varga J., Kemény L., Dékány I.: Investigation of the structure and swelling of poly( $N$-isopropylacrylamide-acrylamide) and poly( $N$-isopropyl-acrylamide-acrylic acid) based copolymer and composite hydrogels. Colloid and Polymer Science, 286, 15751585 (2008).

https://doi.org/10.1007/s00396-008-1933-8

[10] Janovák L., Tallósy S. P., Sztakó M., Deák Á., Bitó T., Buzás N., Bártfai G., Dékány I.: Synthesis of pH-sensitive copolymer thin solid films embedded with silver nanoparticles for controlled release and their fungicide properties. Journal of Drug Delivery Science and Technology, 24, 628-636 (2014). https://doi.org/10.1016/S1773-2247(14)50129-3

[11] Eduok U., Faye O., Szpunar J.: Recent developments and applications of protective silicone coatings: A review of PDMS functional materials. Progress in Organic Coatings, 111, 124-163 (2017). https://doi.org/10.1016/j.porgcoat.2017.05.012 
[12] Levkin P. A., Svec F., Fréchet J. M. J.: Porous polymer coatings: A versatile approach to superhydrophobic surfaces. Advanced Functional Materials, 19, 1993-1998 (2009). https://doi.org/10.1002/adfm.200801916

[13] Kamegawa T., Shimizu Y., Yamashita H.: Superhydrophobic surfaces with photocatalytic self-cleaning properties by nanocomposite coating of $\mathrm{TiO}_{2}$ and polytetrafluoroethylene. Advanced Materials, 24, 36973700 (2012).

https://doi.org/10.1002/adma.201201037

[14] Kim K-D., Seo H. O., Sim C. W., Jeong M-G., Kim Y. D., Lim D. C.: Preparation of highly stable superhydrophobic $\mathrm{TiO}_{2}$ surfaces with completely suppressed photocatalytic activity. Progress in Organic Coatings, 76, 596-600 (2013). https://doi.org/10.1016/j.porgcoat.2012.11.010

[15] Charpentier P. A., Burgess K., Wang L., Chowdhury R. R., Lotus A. F., Moula G.: Nano- $\mathrm{TiO}_{2} /$ polyurethane composites for antibacterial and self-cleaning coatings. Nanotechnology, 23, 425606/1-425606/9 (2012). https://doi.org/10.1088/0957-4484/23/42/425606

[16] Lee J. H., Park E. J., Kim D. H., Jeong M-G., Kim Y. D.: Superhydrophobic surfaces with photocatalytic activity under UV and visible light irradiation. Catalysis Today, 260, 32-38 (2016). https://doi.org/10.1016/j.cattod.2015.05.020

[17] Zhang W., Lu X., Xin Z., Zhou C.: A self-cleaning polybenzoxazine/ $/ \mathrm{TiO}_{2}$ surface with superhydrophobicity and superoleophilicity for oil/water separation. Nanoscale, 7, 19476-19483 (2015).

https://doi.org/10.1039/c5nr06425b

[18] Park E. J., Yoon H. S., Kim D. H., Kim Y. H., Kim Y. D.: Preparation of self-cleaning surfaces with a dual functionality of superhydrophobicity and photocatalytic activity. Applied Surface Science, 319, 367-371 (2014). https://doi.org/10.1016/j.apsusc.2014.07.122

[19] Marmur A., Volpe C. D., Siboni S., Amirfazli A., Drelich J. W.: Contact angles and wettability: Towards common and accurate terminology. Surface Innovations, 5, 3-8 (2017).

https://doi.org/10.1680/jsuin.17.00002

[20] Drelich J.: Guidelines to measurements of reproducible contact angles using a sessile-drop technique. Surface Innovations, 1, 248-254 (2013).

https://doi.org/10.1680/si.13.00010
[21] Chibowski E.: Surface free energy of a solid from contact angle hysteresis. Advances in Colloid and Interface Science, 103, 149-172 (2003). https://doi.org/10.1016/S0001-8686(02)00093-3

[22] Drelich J., Chibowski E.: Superhydrophilic and superwetting surfaces: Definition and mechanisms of control. Langmuir, 26, 18621-18623 (2010).

https://doi.org/10.1021/la1039893

[23] Románszki L., Mohos M., Telegdi J., Keresztes Z., Nyikos L.: A comparison of contact angle measurement results obtained on bare, treated, and coated alloy samples by both dynamic sessile drop and Wilhelmy method. Periodica Polytechnica Chemical Engineering, 58, 53-59 (2014). https://doi.org/10.3311/PPch.7188

[24] Hoffman R.: A study of the advancing interface. I. Interface shape in liquid-gas systems. Journal of Colloid and Interface Science, 50, 228-241 (1975). https://doi.org/10.1016/0021-9797(75)90225-8

[25] Dussan E. B.: On the spreading of liquids on solid surfaces: Static and dynamic contact lines. Annual Review of Fluid Mechanics, 11, 371-400 (1979). https://doi.org/10.1146/annurev.fl.11.010179.002103

[26] Volpe C. D., Siboni S.: The Wilhelmy method: A critical and practical review. Surface Innovations, 6, 120-132 (2018). https://doi.org/10.1680/jsuin.17.00059

[27] Wahl A., Augustynski J.: Charge carrier transport in nanostructured anatase $\mathrm{TiO}_{2}$ films assisted by the selfdoping of nanoparticles. Journal of Physical Chemistry B, 102, 7820-7828 (1998). https://doi.org/10.1021/jp9814000

[28] Krasowska M., Zawala J., Malysa K.: Air at hydrophobic surfaces and kinetics of three phase contact formation. Advances in Colloid and Interface Science, 147148, 155-169 (2009). https://doi.org/10.1016/j.cis.2008.10.003

[29] Janovák L., Deák Á., Tallósy Sz. P., Sebők D., Csapó E., Bohinc K., Abram A., Pálinkó I., Dékány I.: Hydroxyapatite-enhanced structural, photocatalytic and antibacterial properties of photoreactive $\mathrm{TiO}_{2} / \mathrm{HAp} /$ polyacrylate hybrid thin films. Surface and Coatings Technology, 326, 316-326 (2017). https://doi.org/10.1016/j.surfcoat.2017.07.072 